\title{
Modeling thermocapillary migration of a microfluidic droplet on a solid surface
}

\author{
Haihu Liu*, Yonghao Zhang \\ James Weir Fluids Laboratory, Department of Mechanical \& Aerospace Engineering, \\ University of Strathclyde, Glasgow G1 1XJ, UK
}

\begin{abstract}
A multiphase lattice Boltzmann model is developed to simulate immiscible thermocapillary flows with the presence of fluid-surface interactions. In this model, interfacial tension force and Marangoni stress are included by introducing a body force term based on the concept of continuum surface force, and phase segregation is achieved using the recoloring algorithm proposed by Latva-Kokko and Rothman. At a solid surface, fluid-surface interactions are modeled by a partial wetting boundary condition that uses a geometric formulation to specify the contact angle, and a color-conserving boundary closure scheme to improve the numerical accuracy and suppress spurious velocities at the contact line. An additional convection-diffusion equation is solved by the passive scalar approach to obtain the temperature field, which is coupled to the hydrodynamic equations through an equation of state. This model is first validated by simulations of static contact angle and dynamic capillary intrusion process when a constant interfacial tension is considered. It is then used to simulate the thermocapillary migration of a microfluidic droplet on a horizontal solid surface subject to a uniform temperature gradient. We for the first time demonstrate numerically that the droplet motion undergoes two different states depending on the surface wettability: the droplet migrates towards the cooler regions on hydrophilic surfaces but reverses on hydrophobic surfaces. Decreasing the viscosity ratio can enhance the intensity of thermocapillary vortices, leading to an increase in migration velocity. The contact angle hysteresis, i.e., the difference between the advanc-
\end{abstract}

\footnotetext{
*Corresponding author

Email address: haihliu@gmail.com (Haihu Liu)
} 
ing and receding contact angles, is always positive regardless of the contact angle and viscosity ratio. The contact angle hysteresis and the migration velocity both first decreases and then increases with the contact angle, and their minimum values occur at the contact angle of 90 degrees.

Keywords:

Thermocapillary migration, Lattice Boltzmann method, Multiphase flow, Wetting boundary condition, Microfluidics, Droplet dynamics

\section{Introduction}

Thermocapillary convection is a phenomenon of fluid movement that arises as a consequence of the variation of interfacial tension at a fluid-fluid interface caused by temperature gradients. It has been known as a mechanism for driving the motion of droplets and bubbles immersed in a second fluid since the pioneering work of Young et al. [1]. For most fluids interfacial tension is a decreasing function of temperature, and this leads to the movement of droplets or bubbles suspended in a bulk fluid from the regions of lower temperature to the warmer regions; such a subject has been studied extensively due to its importance in space material processing and many other engineering and scientific applications under microgravity conditions where sedimentation and gravity-driven convection are largely eliminated [2]. Over the last decade or so, attention has been focused on using thermocapillary forces to manipulate the motion and dynamic behavior of droplets or bubbles in microfluidic devices, where bulk phenomena can be negligible in comparison with interfacial effects due to large surface-to-volume ratio and low Reynolds number. Thermocapillary actuation is advantageous over convectional hydrodynamic stress, electrohydrodynamic force, and magnetic force methods for droplet and bubble manipulations, as it can be generated easily by means of substrate embedded microheaters $[3,4]$ or by localized laser heating $[5,6]$ that allows contactless, reconfigurable, and real-time control of multiple droplets without the need for any special microfabrication or moving parts. To date, the thermocapillary force has been combined with geometry of the microchannel to realise various droplet manipulations including mixing, sorting, fission, fusion, sampling and switching [7, 8].

Unlike droplets suspended in a carrier fluid, droplets are typically in touch with channel walls in microfluidic devices. The confinement and wettability 
of channel walls would quantitatively or qualitatively modify the physics of thermocapillary migration in a microfluidic channel $[5,9]$. For example, it was observed that a liquid droplet placed on a horizontal solid surface can move toward cooler regions [10], as opposed to that in an unbounded flow condition. There have been a number of experimental studies investigating the migration of a liquid droplet on a solid surface induced by thermal gradients $[11,12,13,14,15]$. However, it is still very difficult to conduct precise experimental measurements of the local temperature and flow fields during the migration process of a droplet. Theoretical studies based on a lubrication approximation have been used for analyzing the migration velocity of a droplet with sufficiently small aspect ratio (defined by the maximum height of the droplet to its length) induced by thermocapillary force $[16,17,10,18]$. Unfortunately, they are unable to solve the transient thermocapillary migration of a spherical cap droplet with a large contact angle due to the limitation of lubrication approximation. Numerical modelling and simulations can complement theoretical and experimental studies, providing an efficient pathway to enhance our understanding of thermocapillary migration of droplets in a microchannel.

Computational modeling of thermocapillary flows with fluid-surface interactions is a challenging task. Discretization errors in computation of interfacial forces may generate unphysical spurious velocities that can cripple the velocity field in the whole computational domain. Minimization of spurious velocities at the interface still remains a major challenge for many numerical models and algorithms. In addition, contact line dynamics is still a challenging problem that has not been fully solved because of its inherent multiscale nature [19]. Finally, due to strong dependence of interfacial tension on temperature, temperature fluctuations result in non-uniform interfacial tension forces and Marangoni stresses that affect the velocity field near the interface, which in turn alters the interfacial temperature distribution through the induced interfacial flows. Although traditional CFD (computational fluid dynamics) methods such as volume-of-fluid [20, 21] and level-set methods $[22,23]$ have been extensively used for simulating various multiphase flow problems, they suffer from numerical instability at the interface region when the interfacial tension becomes a dominant factor in microdroplet behavior [24]. Moreover, an empirical slip model with slip length at the molecular scale has to be introduced in these methods to avoid stress singularities at the moving contact line [25]. Microscopically, the interface between different phases and the contact line dynamics on the solid surface 
are due to interparticle interactions [26]. Thus, mesoscopic level models are expected to describe more accurately the multiscale thermocapillary flows in a confined microfluidic device.

The lattice Boltzmann (LB) method has become a promising alternative to traditional CFD methods for simulating complex fluid flow problems. It is a pseudo-molecular method based on particle distribution functions that performs microscopic operations with mesoscopic kinetic equations and reproduces macroscopic behavior [27]. Its mesoscopic kinetic nature offers many of the advantages of molecular dynamics, making LB method particularly suited for modeling multiphase, multicomponent flows. A number of multiphase, multicomponent models have been proposed in the LB community, which can be classified into four major types: color-fluid model [28, 29, 30, 31], phasefield-based model [32, 33, 34], interparticle-potential model [35, 36, 37], and mean-field theory model [38]. These models have shown great success in modeling multiphase flow problems with a constant interfacial tension [39]. Based on the color-fluid model, we recently proposed the first LB model to simulate thermocapillary flows, through which we first numerically demonstrate that the micro-droplet manipulation can be achieved through the thermocapillary forces induced by the laser heating [40]. Later, we developed two phase-field-based thermocapillary models with one focusing on high-densityratio two-phase flows [41] and the other on modeling fluid-surface interactions [9]. Although the thermocapillary color-fluid model inherits a series of advantages of the model by Halliday and his coworkers [42, 29], such as low spurious velocities, high numerical accuracy and strict mass conservation for each fluid, it can only simulate thermocapillary flows with droplets suspended in a carrier fluid, away from the wall boundary.

In this paper, we present a color-fluid LB model to simulate immiscible thermocapillary flows with the presence of fluid-surface interactions. A twophase collision operator based on the concept of a continuum surface force (CSF) [43] is used to model the interfacial tension force and Marangoni stress, and the recoloring operator proposed by Latva-Kokko and Rothman [44] is introduced to maintain the interface between two fluids. An additional convection-diffusion equation is solved by a passive-scalar approach [45] to obtain the temperature field, which is coupled to the interfacial tension by an equation of state. At the solid surface, a previously developed wetting boundary condition [46] is incorporated into the model to account for fluidsurface interactions, in which the contact angle is enforced through a geometric formulation [47] and the color-conserving boundary closure scheme [48] is 
applied to improve the numerical accuracy and suppress spurious currents at the contact line. The capability and accuracy of this model are first tested by two benchmark cases with analytical solutions. It is then used to simulate the thermocapillary migration of a microfluidic droplet adhering on a horizontal solid surface subject to a uniform temperature gradient, in which the influence of contact angle and fluid viscosity ratio on the droplet migration are systematically investigated.

\section{Methodology}

\subsection{Color-fluid lattice Boltzmann model for thermocapillary flow}

A color-fluid LB model was recently developed by Liu et al. [40] to simulate thermocapillary flows. In this model, red and blue distribution functions $f_{i}^{R}$ and $f_{i}^{B}$ are introduced to represent two different fluids, and the total distribution function is defined as: $f_{i}=f_{i}^{R}+f_{i}^{B}$. Each of the colored fluids undergoes the collision and streaming operations:

$$
f_{i}^{k}\left(\mathbf{x}+\mathbf{e}_{i} \delta_{t}, t+\delta_{t}\right)=f_{i}^{k}(\mathbf{x}, t)+\Omega_{i}^{k}(\mathbf{x}, t),
$$

where the superscript $k=R$ or $B$ denotes the color ("Red" or "Blue"), $f_{i}(\mathbf{x}, t)$ is the distribution function in the $i$-th velocity direction at the position $\mathbf{x}$ and time $t, \mathbf{e}_{i}$ is the lattice velocity in the $i$-th direction, $\delta_{t}$ is the time step, and $\Omega_{i}^{k}$ is the collision operator. The collision operator is the result of the combination of three sub-operators [31]:

$$
\Omega_{i}^{k}=\left(\Omega_{i}^{k}\right)^{(3)}\left[\left(\Omega_{i}^{k}\right)^{(1)}+\left(\Omega_{i}^{k}\right)^{(2)}\right],
$$

where $\left(\Omega_{i}^{k}\right)^{(1)}$ is the Bhatnagar-Gross-Krook (BGK) collision operator, $\left(\Omega_{i}^{k}\right)^{(2)}$ is the two-phase collision operator which contributes to the mixed interfacial region and generates an interfacial force, and $\left(\Omega_{i}^{k}\right)^{(3)}$ represents the recoloring operator which mimics the phase segregation and keeps the interface sharp.

The BGK collision operator is given by

$$
\left(\Omega_{i}^{k}\right)^{(1)}=-\frac{1}{\tau_{f}}\left(f_{i}^{k}-f_{i}^{k, e q}\right),
$$

where $\tau_{f}$ is the dimensionless relaxation time, and $f_{i}^{k, e q}$ is the equilibrium distribution function of $f_{i}^{k}$. Conservation of mass for each fluid and total momentum conservation require

$$
\rho_{k}=\sum_{i} f_{i}^{k}=\sum_{i} f_{i}^{k, e q}
$$




$$
\rho \mathbf{u}=\sum_{k} \sum_{i} f_{i}^{k} \mathbf{e}_{i}=\sum_{k} \sum_{i} f_{i}^{k, e q} \mathbf{e}_{i},
$$

where $\rho_{k}$ is the density of fluid $k, \rho=\rho_{R}+\rho_{B}$ is the total density and $\mathbf{u}$ the local fluid velocity. For the two-dimensional 9-velocity (D2Q9) model [49], the lattice velocity $\mathbf{e}_{i}$ is defined as $\mathbf{e}_{0}=(0,0), \mathbf{e}_{1,3}=( \pm c, 0), \mathbf{e}_{2,4}=(0, \pm c)$, $\mathbf{e}_{5,7}=( \pm c, \pm c)$, and $\mathbf{e}_{6,8}=(\mp c, \pm c)$, where $c=\delta_{x} / \delta_{t}$ is the lattice speed and $\delta_{x}$ the lattice spacing. The equilibrium distribution functions are chosen to respect the conservation constraints of Eqs. (4) and (5)

$$
f_{i}^{k, e q}\left(\rho_{k}, \mathbf{u}\right)=\rho_{k} w_{i}\left[1+\frac{\mathbf{e}_{i} \cdot \mathbf{u}}{c_{s}^{2}}+\frac{\left(\mathbf{e}_{i} \cdot \mathbf{u}\right)^{2}}{2 c_{s}^{4}}-\frac{\mathbf{u}^{2}}{2 c_{s}^{2}}\right],
$$

where $c_{s}=1 / \sqrt{3} c$ is the lattice sound speed, and $w_{i}$ is the weight factor with $w_{0}=4 / 9, w_{1-4}=1 / 9$ and $w_{5-8}=1 / 36$.

An indicator function $\rho^{N}$ is introduced to identify the location of interface, and is defined by

$$
\rho^{N}(\mathbf{x}, t)=\frac{\rho_{r}(\mathbf{x}, t)-\rho_{b}(\mathbf{x}, t)}{\rho_{r}(\mathbf{x}, t)+\rho_{b}(\mathbf{x}, t)}, \quad-1 \leq \rho^{N} \leq 1
$$

In the LBM community, the concept of continuum surface force (CSF) was first used by Lishchuk et al. [42] to model the interfacial force with constant interfacial tension, which was demonstrated to effectively reduce the spurious velocities. It was later extended by Liu and Zhang [40] to model the interfacial force with temperature-dependent interfacial tension and Marangoni stress. Following Liu and Zhang, the interfacial force reads as

$$
\mathbf{F}(\mathbf{x}, t)=\frac{1}{2}\left|\nabla \rho^{N}\right|\left(\sigma \kappa \mathbf{n}+\nabla_{s} \sigma\right)
$$

where $\mathbf{I}$ is the second-order identity tensor, $\nabla_{s}=(\mathbf{I}-\mathbf{n} \otimes \mathbf{n}) \cdot \nabla$ is the surface gradient operator, $\sigma$ is the interfacial tension, $\mathbf{n}$ is the interfacial unit normal vector defined by

$$
\mathbf{n}=\frac{\nabla \rho^{N}}{\left|\nabla \rho^{N}\right|},
$$

and $\kappa$ is is the local interface curvature related to $\mathbf{n}$ by

$$
\kappa=-\nabla_{s} \cdot \mathbf{n} .
$$


In a thermocapillary flow, an equation of state is needed to relate the interfacial tension to the temperature, which may be linear or nonlinear. For the sake of simplicity, we only consider a linear relation between the interfacial tension and the temperature, i.e.,

$$
\sigma(T)=\sigma_{r e f}+\sigma_{T}\left(T-T_{r e f}\right),
$$

where $\sigma_{r e f}$ is the interfacial tension at the reference temperature $T_{r e f}$ and $\sigma_{T}$ is the interfacial tension gradient with respect to temperature.

Substituting of Eqs. (9) and (11) into Eq. (8), we obtain the interfacial force as

$$
\mathbf{F}(\mathbf{x}, t)=\frac{1}{2} \sigma \kappa \nabla \rho^{N}+\frac{1}{2} \sigma_{T}\left|\nabla \rho^{N}\right|(\mathbf{I}-\mathbf{n} \otimes \mathbf{n}) \cdot \nabla T .
$$

The interfacial force is then incorporated into LBM through the body force model of Guo et al. [50] because of its high accuracy in modeling a spatially varying body force and capability in reducing effectively the spurious velocities [29, 46]. According to Guo et al. [50], the two-phase collision operator $\left(\Omega_{i}^{k}\right)^{(2)}$ is written as

$$
\left(\Omega_{i}^{k}\right)^{(2)}=A_{k}\left(1-\frac{1}{2 \tau_{f}}\right) \omega_{i}\left[\frac{\mathbf{e}_{i}-\mathbf{u}}{c_{s}^{2}}+\frac{\mathbf{e}_{i} \cdot \mathbf{u}}{c_{s}^{4}} \mathbf{e}_{i}\right] \cdot \mathbf{F},
$$

where the free parameter $A_{k}$ satisfies $\sum_{k} A_{k}=1$, and the velocity is redefined to include some of the effect of external body force [50]

$$
\rho \mathbf{u}=\sum_{k} \sum_{i} f_{i}^{k} \mathbf{e}_{i}+\frac{1}{2} \mathbf{F} \delta_{t} .
$$

Using the Chapman-Enskog multiscale analysis, Eq. (1) can be reduced to the Navier-Stokes equations in the low frequency, long wavelength limit with Eqs. (3), (6), (13) and (14). The resulting equations are

$$
\begin{gathered}
\partial_{t} \rho+\nabla \cdot(\rho \mathbf{u})=0, \\
\partial_{t}(\rho \mathbf{u})+\nabla \cdot(\rho \mathbf{u u})=-\nabla p+\nabla \cdot\left[\rho \nu\left(\nabla \mathbf{u}+\nabla \mathbf{u}^{T}\right)\right]+\mathbf{F},
\end{gathered}
$$

where $p=\rho c_{s}^{2}$ is the pressure, and $\nu=c_{s}^{2}\left(\tau_{f}-\frac{1}{2}\right) \delta_{t}$ is the kinematic viscosity of the fluid mixture. To account for unequal viscosities of the two fluids, the following function is used to determine the viscosity of the fluid mixture [51]

$$
\frac{1}{\nu}=\frac{1+\rho^{N}}{2 \nu_{R}}+\frac{1-\rho^{N}}{2 \nu_{B}} \quad \text { or } \quad \frac{1}{\tau-0.5}=\frac{1+\rho^{N}}{2\left(\tau_{R}-0.5\right)}+\frac{1-\rho^{N}}{2\left(\tau_{B}-0.5\right)},
$$


where $\nu_{k}(k=R$ or $B)$ is the kinematic viscosity of fluid $k$, which is related to the dimensionless relaxation time $\tau_{k}$ as $\nu_{k}=c_{s}^{2}\left(\tau_{k}-\frac{1}{2}\right) \delta_{t}$. It has been demonstrated that the choice of Eq. (17) can ensure the continuity of viscosity flux across the interface [51].

To minimize the discretization errors, the derivatives in Eq. (12) are evaluated numerically through the nine-point isotropic finite difference stencils for a variable $\psi[31]$ :

$$
\nabla \psi(\mathbf{x}, t)=\frac{1}{c_{s}^{2}} \sum_{i} w_{i} \psi\left(\mathbf{x}+\mathbf{e}_{i} \delta_{t}, t\right) \mathbf{e}_{i}
$$

Although the two-phase collision operator generates the interfacial tension and Marangoni stress, it does not guarantee the immiscibility of both fluids. To promote phase segregation and maintain the interface, the recoloring algorithm proposed by Latva-Kokko and Rothman [44] is applied. This algorithm allows the red and blue fluids to mix moderately at the tangent of the interface, and at the same time keeps the color distribution symmetric with respect to the color gradient. Thus, it helps further reduce the spurious velocities and removes the lattice pinning problem produced by the original recoloring algorithm of Gunstensen et al. [52]. In addition, compared to the recoloring algorithm of Gunstensen et al., it greatly increases the rate of convergence, improves the numerical stability and accuracy of the solutions over a broad range of model parameters [52]. Following Latva-Kokko and Rothman [44], the recoloring operators for the red and blue fluids are defined by

$$
\begin{aligned}
& \left(\Omega_{i}^{R}\right)^{(3)}\left(f_{i}^{R}\right)=\frac{\rho_{R}}{\rho} f_{i}^{\dagger}+\beta \frac{\rho_{R} \rho_{B}}{\rho} w_{i}\left|\mathbf{e}_{i}\right| \cos \left(\varphi_{i}\right), \\
& \left(\Omega_{i}^{B}\right)^{(3)}\left(f_{i}^{B}\right)=\frac{\rho_{B}}{\rho} f_{i}^{\dagger}-\beta \frac{\rho_{R} \rho_{B}}{\rho} w_{i}\left|\mathbf{e}_{i}\right| \cos \left(\varphi_{i}\right),
\end{aligned}
$$

where $f_{i}^{\dagger}$ denotes the total distribution function after the two-phase collision. $\beta$ is the segregation parameter and is fixed at 0.7 to maintain a narrow interface thickness and reduce spurious velocities [53]; several recent studies $[54,31]$ also showed that this choice is necessary to reproduce correct interface dynamics. $\varphi_{i}$ is the angle between the indicator function gradient $\nabla \rho^{N}$ and the lattice vector $\mathbf{e}_{i}$, which is defined by

$$
\cos \left(\varphi_{i}\right)=\frac{\mathbf{e}_{i} \cdot \nabla \rho^{N}}{\left|\mathbf{e}_{i}\right|\left|\nabla \rho^{N}\right|}
$$


To model the temperature field evolution, another particle distribution function $g_{i}$ is used, with the governing equation [45, 40]

$$
g_{i}\left(\mathbf{x}+\mathbf{e}_{i} \delta_{t}, t+\delta_{t}\right)-g_{i}(\mathbf{x}, t)=-\frac{1}{\tau_{g}}\left[g_{i}(\mathbf{x}, t)-g_{i}^{e q}(\mathbf{x}, t)\right]
$$

where $\tau_{g}$ is the relaxation parameter, and $g_{i}^{e q}$ is the equilibrium distribution function

$$
g_{i}^{e q}=T \omega_{i}\left[1+\frac{\mathbf{e}_{i} \cdot \mathbf{u}}{c_{s}^{2}}+\frac{\left(\mathbf{e}_{i} \cdot \mathbf{u}\right)^{2}}{2 c_{s}^{4}}-\frac{\mathbf{u}^{2}}{2 c_{s}^{2}}\right],
$$

where $T$ is the temperature. With the macroscopic velocity given by Eq. (14), Eq. (21) can recover the following convection-diffusion equation

$$
\partial_{t} T+\mathbf{u} \cdot \nabla T=\nabla \cdot(\kappa \nabla T)
$$

where $\kappa=\left(\tau_{g}-\frac{1}{2}\right) c_{s}^{2} \delta_{t}$ is the thermal conductivity. Following the definition of fluid viscosity given by Eq. (17), we define the thermal conductivity of mixture as

$$
\frac{1}{\kappa}=\frac{1+\rho^{N}}{2 \kappa_{R}}+\frac{1-\rho^{N}}{2 \kappa_{B}},
$$

where $\kappa_{R}\left(\kappa_{B}\right)$ is the thermal conductivity of red (blue) fluid.

\subsection{Wetting boundary condition}

To model fluid-surface interactions, our recently developed wetting boundary condition (WBC) [46] is adopted because of its advantages in modeling the contact line dynamics such as high accuracy and small spurious velocities. In the WBC, a constant (microscale) contact angle is prescribed at the solid surface, which is assumed to be equal to the equilibrium contact angle $\theta^{e q}$, as done by Ding and Spelt $[47,55]$. The contact angle is enforced by using the geometrical properties of the indicator function $\rho^{N}$ at the contact line, a color-conserving boundary closure scheme [48] is applied to ensure mass conservation for each fluid, and a variant of the recoloring operator is designed to maintain the reasonable interface at the solid boundary.

Assuming that the contours of the indicator function in the diffuse interface are approximately parallel to each other, the equilibrium contact angle $\theta^{e q}$ can be calculated geometrically in terms of $\rho^{N}$ by [47]

$$
\tan \left(\frac{\pi}{2}-\theta^{e q}\right)=\frac{-\mathbf{n}_{w} \cdot \nabla \rho^{N}}{\left|\nabla \rho^{N}-\left(\mathbf{n}_{w} \cdot \nabla \rho^{N}\right) \mathbf{n}_{w}\right|}=\frac{-\mathbf{n}_{w} \cdot \nabla \rho^{N}}{\left|\left(\mathbf{t}_{w} \cdot \nabla \rho^{N}\right) \mathbf{t}_{w}\right|},
$$




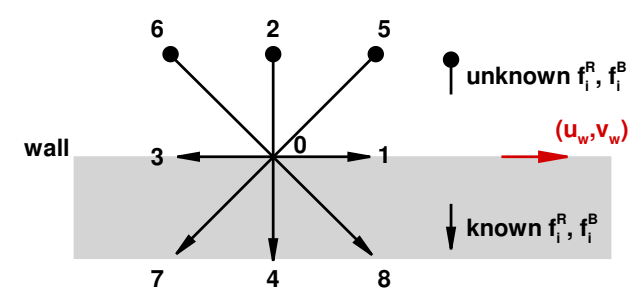

Figure 1: (Color online) Illustration of a D2Q9 lattice node on the bottom wall of a twodimensional domain after the propagation step. The wall is supposed to move with the velocity $\mathbf{u}_{w}=\left(u_{w}, v_{w}\right)$. The fluid and solid are represented by the white and grey regions, respectively.

where $\mathbf{n}_{w}$ is the unit normal vector to wall pointing towards the fluid, and $\mathbf{t}_{w}$ is the unit vector tangential to wall. From Eq. (25), one has

$$
\mathbf{n}_{w} \cdot \nabla \rho^{N}=-\Theta_{w}\left|\mathbf{t}_{w} \cdot \nabla \rho^{N}\right|
$$

with

$$
\Theta_{w}=\tan \left(\frac{\pi}{2}-\theta^{e q}\right)
$$

In the present algorithm, the tangential component of $\nabla \rho^{N}$ is determined by the central difference approximation. Then, the normal component of $\nabla \rho^{N}$ is obtained using Eq. (26). Thus, the contact angle is implicitly imposed by the gradient of indicator function at the solid wall.

The color-conserving boundary closure scheme [48] is employed to determine appropriate distribution functions for live links after the two-phase collision. Fig. 1 depicts a lattice node on the bottom wall, which moves at a velocity of $\mathbf{u}_{w}=\left(u_{w}, v_{w}\right)$. Assume that the node lies in the interface between the red and blue fluids. At this lattice node, the post-propagation value of distribution function $f_{i}^{k}$ exists only for $i \neq 2,5,6$, thus, the total of distribution functions that propagate into the fluid domain at the node depicted for each fluid is given by $\varrho_{i n}^{k}=\sum_{i \neq 2,5,6} f_{i}^{k}$. After the two-phase collision, the distribution functions need to be considered only for live links, i.e., $i \neq 4,7,8$ since the distribution functions with $i=4,7,8$ will propagate out of the fluid domain after the propagation. Therefore, the effective mass for each fluid after two-phase collision is given as $\sum_{i \neq 4,7,8} f_{i}^{k \dagger}$. To ensure mass conservation for each fluid during the collision, it requires that,

$$
\sum_{i \neq 4,7,8} f_{i}^{k \dagger}=\varrho_{i n}^{k}
$$


According to Eq. (1), the distribution function for the fluid $k$ after the two-phase collision can be written as

$$
f_{i}^{k \dagger}=f_{i}^{k, e q}\left(\rho_{k}^{*}, \mathbf{u}_{w}\right)+\left(\Omega_{i}^{k}\right)^{(2)}+\left(1-\frac{1}{\tau_{f}}\right) f_{i}^{k(1)},
$$

where $f_{i}^{k(1)}=f_{i}^{k}-f_{i}^{k, e q}$ denotes the higher-order component of the distribution function $f_{i}^{k}$, and $\rho_{k}^{*}$ is an auxiliary boundary density. The subtotal of $f_{i}^{k(1)}$ on the live links is assumed to be zero, and the subtotal of the two-phase collision operator on the live links disrupts the conservation by

$$
\Delta M_{k}=\sum_{i \neq 4,7,8}\left(\Omega_{i}^{k}\right)^{(2)}=\sum_{i \neq 4,7,8} A_{k}\left(1-\frac{1}{2 \tau_{f}}\right) \omega_{i}\left[\frac{\mathbf{e}_{i}-\mathbf{u}}{c_{s}^{2}}+\frac{\mathbf{e}_{i} \cdot \mathbf{u}}{c_{s}^{4}} \mathbf{e}_{i}\right] \cdot \mathbf{F} \neq 0 .
$$

By substituting of Eqs. (29) and (30) into Eq. (28), the auxiliary boundary density is obtained

$$
\rho_{k}^{*}=\frac{\varrho_{i n}^{k}-\Delta M_{k}}{\sum_{i \neq 4,7,8} f_{i}^{k, e q}\left(1, \mathbf{u}_{w}\right)}=\frac{\varrho_{i n}^{k}-\Delta M_{k}}{\frac{5+3 v_{w}-3 v_{w}^{2}}{6}} .
$$

The higher-order component $f_{i}^{k(1)}$ should satisfy the following constraints as given in Refs. [48]

$$
\begin{aligned}
\sum_{i \neq 4,7,8} f_{i}^{k(1)} & =0, \\
\sum_{i} f_{i}^{k(1)} e_{i \alpha} & =\frac{1}{2} F_{\alpha}, \\
\sum_{i} f_{i}^{k(1)} e_{i \alpha} e_{i \beta} & =-2 \rho_{k}^{*} c_{s}^{2} \tau_{f} S_{\alpha \beta},
\end{aligned}
$$

where $S_{\alpha \beta}$ is the strain rate tensor and defined as

$$
S_{\alpha \beta}=\frac{1}{2}\left(\partial_{\alpha} u_{\beta}+\partial_{\beta} u_{\alpha}\right)+\frac{\delta_{t}}{4 \rho c_{s}^{2} \tau_{f}}\left(F_{\alpha} u_{\beta}+F_{\beta} u_{\alpha}\right) .
$$

Solving the under-specified system of equations, i.e., Eq. (32), through the singular value decomposition (SVD) method, one obtains the solution of 
$f_{i}^{k(1)}$ on live links [46]

$$
\left(\begin{array}{l}
f_{0}^{k(1)} \\
f_{1}^{k(1)} \\
f_{2}^{k(1)} \\
f_{3}^{k(1)} \\
f_{5}^{k(1)} \\
f_{6}^{k(1)}
\end{array}\right)=\frac{1}{36}\left(\begin{array}{ccccc}
0 & -5 & -12 & -2 & 0 \\
3 & -2 & 6 & -8 & 0 \\
0 & 1 & -12 & 10 & 0 \\
-3 & -2 & 6 & -8 & 0 \\
3 & 4 & 6 & 4 & 9 \\
-3 & 4 & 6 & 4 & -9
\end{array}\right)\left(\begin{array}{c}
\delta_{t} F_{x} \\
\delta_{t} F_{y} \\
\omega S_{x x} \\
\omega S_{y y} \\
\omega S_{x y}
\end{array}\right)
$$

where $\omega=-2 \rho_{k}^{*} c_{s}^{2} \tau_{f}$.

Once the distribution functions after the two-phase collision are obtained by Eq. (29), a modified recoloring step is required for the boundary node to maintain the correct interface. Based on the color conservation, the postsegregation distribution functions assigned to the live links should satisfy [48]

$$
\sum_{i \neq 4,7,8} f_{i}^{R \ddagger}=\varrho_{i n}^{R}, \quad \sum_{i \neq 4,7,8} f_{i}^{B \ddagger}=\varrho_{i n}^{B}, \quad \sum_{i \neq 4,7,8} f_{i}^{\ddagger}=\varrho_{i n}^{R}+\varrho_{i n}^{B},
$$

where the superscript ' $f$ ' represents the post-segregation distribution function.

We define $\rho_{R}$ and $\rho_{B}$ as the boundary node red and blue densities that will satisfy mass conservation, and $\rho$ as the total density. To obtain an equation of $\rho_{R}$ or $\rho_{B}$, we substitute Eq. (19) into Eq. (35) and have

$$
\frac{\rho_{R}}{\rho_{R}+\rho_{B}}\left(\varrho_{i n}^{R}+\varrho_{i n}^{B}\right)+\beta \frac{\rho_{R} \rho_{B}}{\rho_{R}+\rho_{B}} \mathbf{n} \cdot \sum_{i \neq 4,7,8}\left(w_{i} \mathbf{e}_{i}\right)=\varrho_{i n}^{R},
$$

which can be simplified as

$$
\frac{\rho_{R}}{\rho_{R}+\rho_{B}}\left(\varrho_{i n}^{R}+\varrho_{i n}^{B}\right)+\beta \frac{\rho_{R} \rho_{B}}{\rho_{R}+\rho_{B}}\left(\frac{1}{6} n_{y}\right)=\varrho_{i n}^{R},
$$

where $n_{y}$ is the $y$-component of the interfacial normal vector $\mathbf{n}$. The conservation of total mass requires

$$
\rho=\sum_{k} \rho_{k}^{*}
$$

Combining Eq. (37) and Eq. (38), we obtain a quadratic equation with respect to $\rho_{R}$ :

$$
\beta n_{y} \rho_{R}^{2}-\left[6\left(\varrho_{i n}^{R}+\varrho_{i n}^{B}\right)+\beta n_{y} \rho\right] \rho_{R}+6 \rho \varrho_{i n}=0,
$$




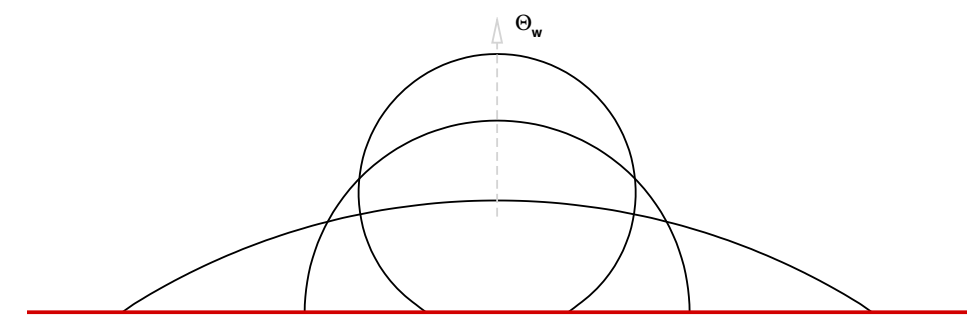

Figure 2: (Color online) Equilibrium droplet shapes obtained through adjusting the dimensionless parameter $\Theta_{w}$ at $M=10$. The values of $\Theta_{w}$ are taken as $\sqrt{3}, 0$, and $-\sqrt{3}$ along the direction of arrow.

which can be solved numerically through the Newton-Raphson method. Then, $f_{i}^{R \ddagger}$ can be calculated by the use of the following segregation formula

$$
f_{i}^{R \ddagger}=\frac{\rho_{R}}{\rho_{R}+\rho_{B}} f_{i}^{\dagger}+\beta \frac{\rho_{R} \rho_{B}}{\rho_{R}+\rho_{B}} w_{i} \mathbf{e}_{i} \cdot \mathbf{n} .
$$

Finally, it is worth noting that the present model is a diffuseinterface model with finite interface thickness. Although a no-slip condition is used at the solid boundary, the motion of contact lines naturally arise as a result of the diffusive flow that occurs in the diffuse interface region.

\section{Results and discussion}

\subsection{Model validation}

In Ref. [40], the present color-fluid LB model has been validated to be able to accurately simulate thermocapillary flows with absence of fluid-surface interactions. Here we focus on examining its extended capability of modeling fluid-surface interactions with variable viscosity ratio through two benchmark cases: static contact angle simulations and a dynamic capillary intrusion test.

First, we perform static contact angle simulations in a $200 \times 100$ lattice domain. The dependence of the interfacial tension on the temperature is switched off so that a constant interfacial tension is used. A semi-circular stationary droplet (red fluid) with the radius $R=25$ initially sits along the center line on the bottom wall. The periodic boundary condition is used in the horizontal direction while the wetting boundary condition, which is given in Subsection 2.2, is imposed at top and bottom walls in the vertical direction. Pooley et al. [56] used the phase-field-based (i.e., free-energy) model 


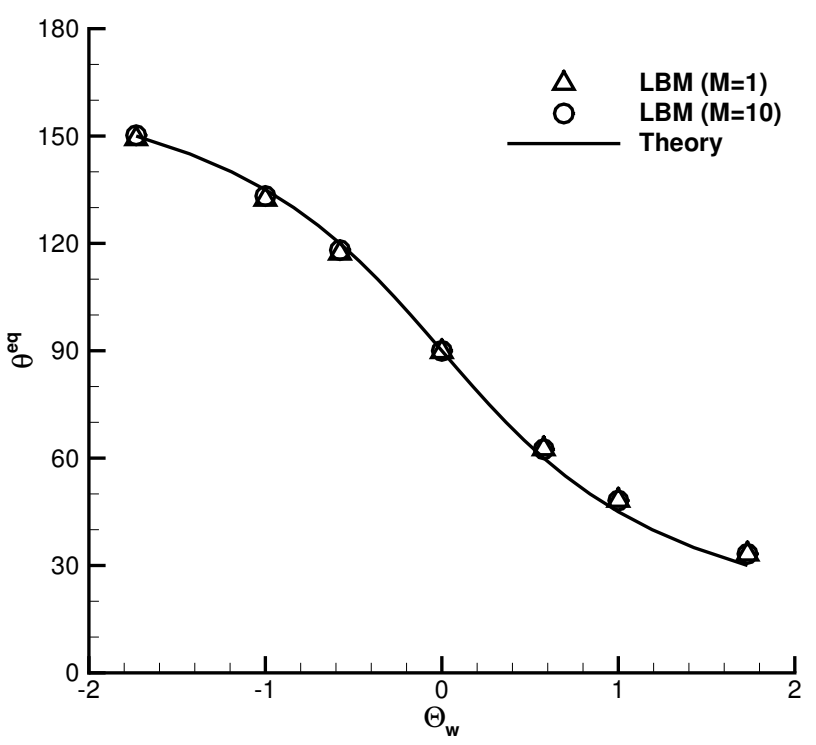

Figure 3: Contact angle as a function of $\Theta_{w}$ for the viscosity ratios $M=1$ (represented by triangles) and 10 (represented by circles). The solid line represents the theoretical predictions by Eq. (27). 
to simulate fluid-surface interactions and identified that the BGK model produces incorrect results for the equilibrium contact angle due to strong spurious velocities when the binary fluids have different viscosities. To examine whether this also happens to the present model, two different viscosity ratios $\left(M=\frac{\nu_{R}}{\nu_{B}}\right)$ are investigated: (a) $M=1$ with $\nu_{R}=\nu_{B}=0.2$, and (b) $M=10$ with $\nu_{R}=0.35$ and $\nu_{B}=0.035$. The other parameters are fixed as $\rho_{R}=\rho_{B}=1$ and $\sigma=0.02$. Each simulation is run until the shape of droplet does not change, i.e., reaching an equilibrium state. Different contact angles can be achieved through adjusting the dimensionless parameter $\Theta_{w}$ according to Eq. (26). Fig. 2 shows equilibrium shapes of the droplet with $\Theta_{w}=\sqrt{3}, 0$ and $-\sqrt{3}$ for the viscosity ratio $M=10$. Their corresponding equilibrium contact angles, calculated from the measured droplet height and base diameter, are $33.1^{\circ}, 89.7^{\circ}$ and $149.2^{\circ}$, respectively. The simulated equilibrium contact angle as a function of $\Theta_{w}$ for $M=1$ and $M=10$ is presented in Fig. 3. It is clearly observed that the simulation results are independent of the viscosity ratio, and are in good agreement with the theoretical solution, Eq. (27), in the range of contact angles from $30^{\circ}$ to $150^{\circ}$. A numerical artifact observed in many numerical methods is the presence of spurious velocities at the phase interface. This is also true in our case. Table 1 shows the maximum spurious velocities $\left(|\mathbf{u}|_{\max }\right)$ at various $\theta^{e q}$ for $M=1$ and 10, where the values of $|\mathbf{u}|_{\max }$ are magnified by $10^{4}$ times. For all of the cases considered, the maximum spurious velocities are of order $O\left(10^{-4}\right)$ or even smaller, comparable to those produced by a recently improved color-fluid model in the stationary bubble test [31], where the bubble is immersed in an infinite domain. Several techniques have been proposed to reduce unwanted spurious velocities in multiphase LB models, and they include increasing the isotropy order of the interfacial force [57], improving the force scheme $[58,59,60]$, and using the multiplerelaxation-time (MRT) model instead of the BGK model $[61,59,9]$. Discussion of their usefulness in the present model is beyond the scope of this paper.

Capillary intrusion, which originated from the pioneering work of Washburn [62], provides a good benchmark for assessing whether a multiphase model is able to simulate moving contact line problems. The velocity of a wetting fluid column intruding a horizontal capillary tube, as shown in Fig. 4, is determined by the balance between the pressure difference over the interface, the Laplace pressure, and the viscous drag of the intruding fluid. If 
Table 1: The maximum spurious velocities $\left(|\mathbf{u}|_{\max }\right)$ at various $\theta^{e q}$ for $M=1$ and 10. All angles are shown in degrees.

\begin{tabular}{|c|c|c|c|c|c|c|c|c|}
\hline \multicolumn{2}{|c|}{$\overline{\theta^{e q}}$} & 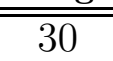 & $\overline{455}$ & $\overline{\overline{660}}$ & $\overline{90}$ & 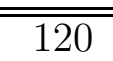 & 135 & $\overline{150}$ \\
\hline | & $M=1$ & 3.692 & 4.810 & 4.210 & 0.537 & 2.710 & 2.716 & 3.852 \\
\hline$|\mathbf{u}|_{\max } \times 10^{-}$ & $M=10$ & 1.991 & 2.893 & 2.664 & 0.086 & 2.028 & 1.622 & 2.536 \\
\hline
\end{tabular}

Figure 4: (Color online) Simulation setup for capillary intrusion. The intruding (red) fluid is the wetting phase while the defending (blue) fluid is the non-wetting phase. The portion in the center of the domain is a capillary tube of length $L$.

the gravity and inertial effects are neglected, this balance can be expressed as $[63,64]$

$$
\sigma \cos (\theta)=\frac{6}{r}\left[\eta_{R} x+\eta_{B}(L-x)\right] \frac{d x}{d t},
$$

where $\theta$ is the contact angle, $r$ is the capillary tube width, $x$ is the position of the phase interface, and $\eta_{R}$ and $\eta_{B}$ are the dynamic viscosities of the red (wetting) and blue (non-wetting) fluids, respectively. In the analytical derivation, $\theta$ is specified as a wall boundary condition. However, for comparison with simulations or experiments, $\theta$ should be taken as the dynamic contact angle [63, 64]. The system consists of a $400 \times 25$ lattice domain with periodic boundary conditions used in the $x$ direction. In the middle portion the boundaries of the capillary tube are no-slip and wetting. The length of the capillary tube is taken as $L=200$ lattices. Outside of the middle portion, the boundary conditions are periodic in the $y$ direction. We run the simulations with the parameters $\rho_{R}=\rho_{B}=1, \sigma=5 \times 10^{-3}$ and $r=15$ for two different viscosity ratios: (a) $M=1$ and (b) $M=10$. The equilibrium contact angle is chosen as $\theta^{e q}=45^{\circ}$ to represent hydrophilic capillary tube for the intruding red fluid. Fig. 5 shows the comparison between our simulation results and the analytical predictions from Eq. (41) for $M=1$ and 10. Note that Eq. (41) is plotted using the dynamic contact angle, measured from our LB simulations. For $M=1$ and $M=10$, the measured dynamic contact angles are respectively $\theta=52.23^{\circ}$ and $52.69^{\circ}$, which are very close but deviate from $\theta^{e q}$. It can be seen that our developed color-fluid model can predict Eq. (41) reasonably well for variable viscosity ratios. 

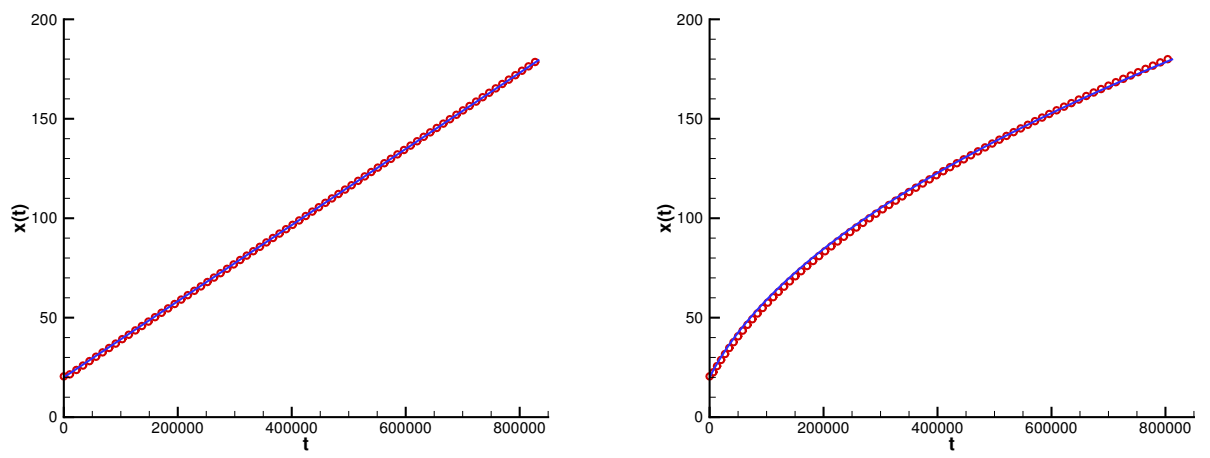

Figure 5: (Color online) The length of the column of the intruding fluid, $x(t)$, as a function of time for the viscosity ratios of (a) $M=1$ and (b) $M=10$. The (red) open circles represent simulation results and the (blue) solid lines are the theoretical predictions from Eq. (41).

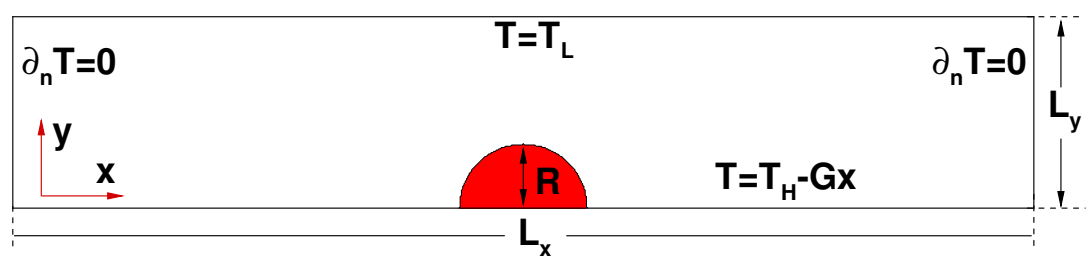

Figure 6: Schematic diagram of a liquid droplet on a solid substrate subject to a uniform temperature gradient. The upper wall is set to be a constant temperature $T_{L}$ and the adiabatic boundary conditions are imposed for the sidewalls. A semi-circular droplet is initially placed on the bottom substrate with its center located at $\left(L_{x} / 2,0\right)$.

\subsection{Thermocapillary migration of a microdroplet on a horizontal solid sub- strate}

In this section, the developed color-fluid LB model is applied to numerically study thermocapillary migration of a liquid droplet on a horizontal solid surface subject to a uniform temperature gradient.

At first, we give a brief description of the problem setup. As shown in Fig. 6, a stationary semicircular droplet of radius $R=50$ is initially placed on a smooth substrate at the bottom. The computational domain size is $L_{x} \times L_{y}=16 R \times 3 R$ and the initial droplet center is $\left(x_{0}, y_{0}\right)=(8 R, 0)$. All boundaries of the computational domain are considered as stationary walls with no-slip conditions. A linear temperature profile is imposed on the bottom wall, i.e., $T(x, 0)=T_{H}-G \times x$, which satisfies $T=T_{H}$ at $x=0$ 
and $T=T_{L}$ at $x=L_{x}$. A constant temperature $T=T_{L}$ is specified on the top wall and the adiabatic boundary conditions are applied for the sidewalls, i.e., $\partial_{n} T=0$. We follow the method of Liu et al. [65] to implement these temperature boundary conditions. To account for the effect of temperature on interfacial tension, Eq. (11) is used with $\sigma_{T}=-4 \times 10^{-4}, \sigma_{\text {ref }}=4.5 \times 10^{-2}$, and $T_{r e f}=T_{L}=0$. Besides, we choose $T_{H}=80$ so that the horizontal temperature gradient $G=0.1$ on the bottom wall.

The thermocapillary migration can be characterized by several important dimensionless parameters: Reynolds number $(R e)$, Marangoni number $(M a)$, capillary number $(\mathrm{Ca})$, and the ratios of viscosity $(\mathrm{M})$ and thermal conductivity $(\chi)$ of fluids. By choosing the blue fluid as the continuous phase, these dimensionless parameters are defined as

$$
R e=\frac{R U}{\nu_{B}}, \quad M a=\frac{R U}{\kappa_{B}}, \quad C a=\frac{U \rho \nu_{B}}{\sigma_{r e f}}, \quad \chi=\frac{\kappa_{R}}{\kappa_{B}},
$$

where $U=-\frac{\sigma_{T} G R}{\rho \nu_{B}}$ is the characteristic velocity of the system. Note that the viscosity ratio $M$ has been defined in Subsection 3.1. In addition, the surface wettability plays an important role in determining the thermocapillary migration process in a microchannel due to large surface-to-volume ratio, which is described by the equilibrium contact angle $\theta^{e q}$. In this study, the influence of surface wettability and $M$ on droplet dynamic behavior will be investigated for constant $R e, C a, M a$ and $\chi$, unless otherwise stated, which are fixed at $10,4.4 \times 10^{-2}, 10$, and 1 , respectively.

The influence of contact angle is first investigated for a fixed viscosity ratio, i.e., $M=1$. Five different contact angles are used in the simulations, i.e., $\theta^{e q}=30^{\circ}, 60^{\circ}, 90^{\circ}, 120^{\circ}$ and $150^{\circ}$, which are achieved through adjusting the value of $\Theta_{w}$ whilst keeping the other parameters fixed. For all contact angles under consideration, the self-motion of the droplet is driven by the thermo-induced interfacial tension gradient on the substrate. However, the droplet motion can undergo two different states, depending on the value of $\theta^{e q}$ : the droplet migrates from the region of high temperature to the colder region on hydrophilic surfaces, i.e., $\theta^{e q}=30^{\circ}$ and $60^{\circ}$; and the droplet migrates from the region of low temperature to the warmer region on neutral and hydrophobic surfaces, i.e., $\theta^{e q} \geq 90^{\circ}$. The two states can be observed in Fig. 7 (since the droplet position has changed from its initial value $\left(x_{0}, y_{0}\right)$ ), which plots the flowfield (including the velocity vectors and streamlines) and the corresponding temperature field surrounding the moving droplet for various $\theta^{e q}$ at $t=2 \times 10^{5}$. The two states can be explained by a simple 
(a)
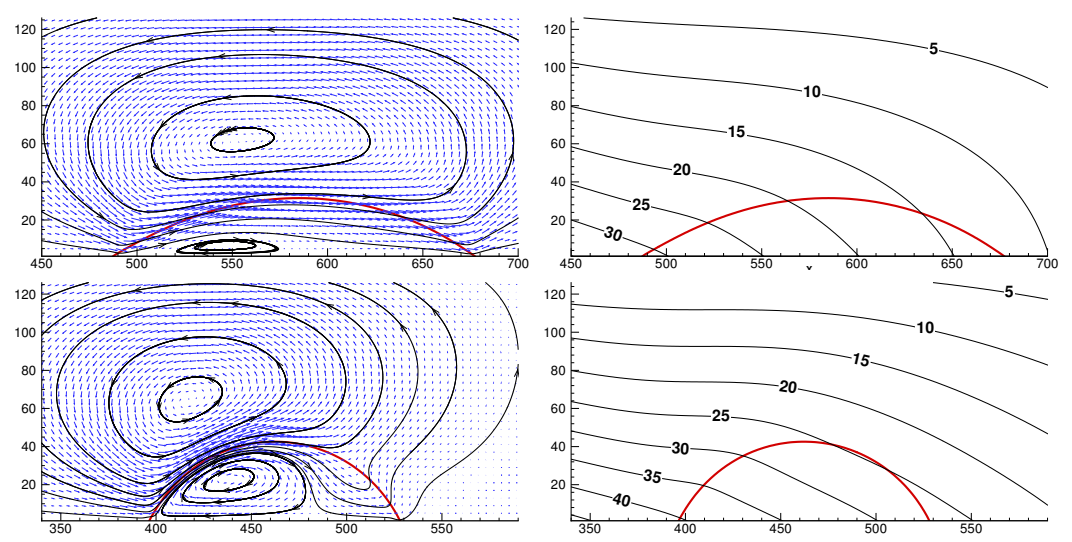

(b)

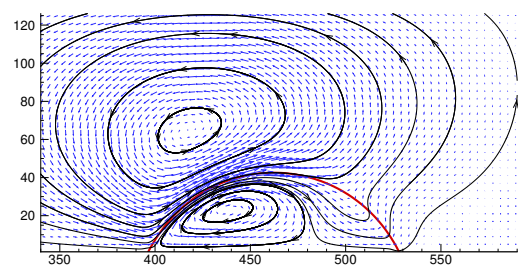

(c)
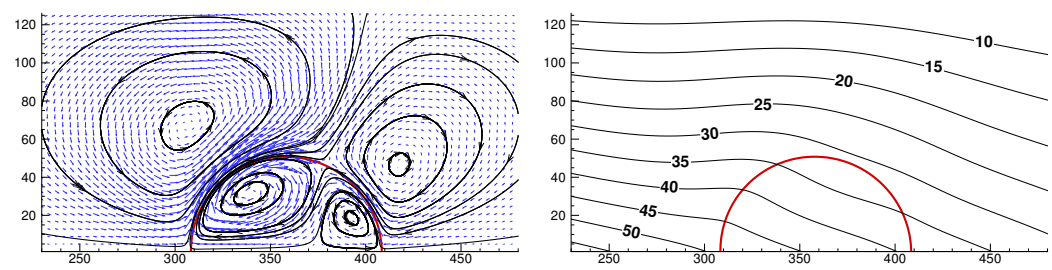

(d)
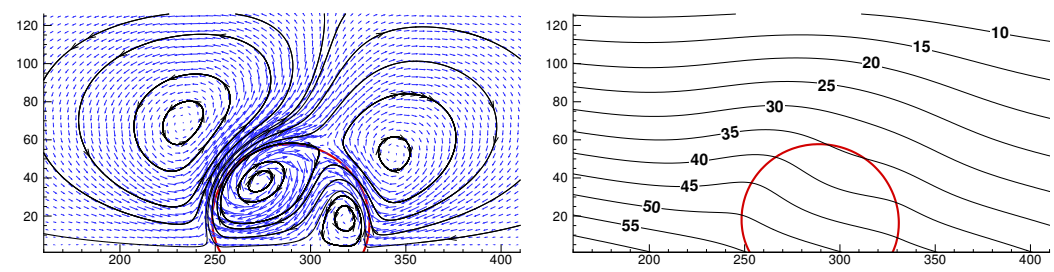

(e)
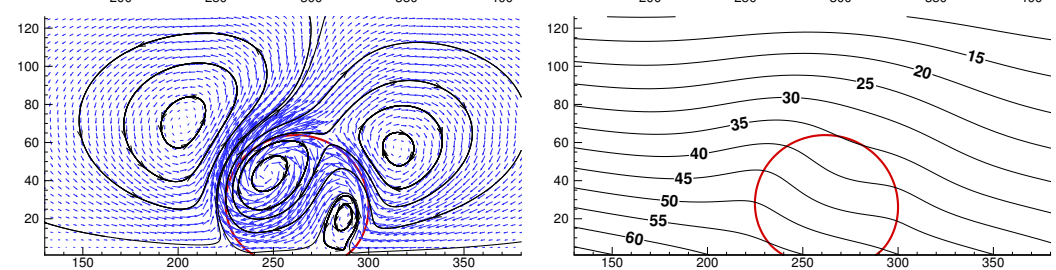

Figure 7: (Color online) The flow field (the left plane) and the temperature field (the right plane) surrounding the moving droplet for $M=1$ at the contact angle: (a) $30^{\circ}$, (b) $60^{\circ}$, (c) $90^{\circ}$, (d) $120^{\circ}$ and (e) $150^{\circ}$. The red lines are zero contours of $\rho^{N}$, the blue lines with arrows are the velocity vectors, and the black lines with arrows are the streamlines. 
force balance between the shear stresses exerted by the solid surface on the droplet and the pressure difference from thermally induced interfacial tension gradient, which is given by [66]

$$
\left(\sigma_{A} \cos \theta_{A}-\sigma_{R} \cos \theta_{R}\right)+s_{w}=0,
$$

where $s_{w}$ is the integration of shear stresses along the droplet/solid interface, and the subscripts $A$ and $R$ refer to the advancing and receding sides of the moving droplet. Since the shear force prevents the relative motion of the droplet, we have $s_{w}<0$, which leads to $\sigma_{A} \cos \theta_{A}>\sigma_{R} \cos \theta_{R}$. We further assume that the contact angle hysteresis is small, i.e., $\theta_{A} \approx \theta_{R}$, which will be demonstrated below. Thus, for hydrophilic surfaces we can obtain $\sigma_{A}>\sigma_{R}$, implying that the droplet moves towards the region with low temperature. Conversely, the droplet moves towards the region with high temperature for hydrophobic surfaces because $\sigma_{A}<\sigma_{R}$.

As shown in the left column of Fig. 7, the vortices can be clearly seen surrounding the droplet for all $\theta^{e q}$ but exhibit different features. For the lowest contact angle, i.e., $\theta^{e q}=30^{\circ}$ a small vortex with clockwise rotation is observed at the bottom wall near the left meniscus inside the droplet. Meanwhile, an external vortex appears on the top of the droplet. When $\theta^{e q}$ increases to $60^{\circ}$, the internal vortex grows which can almost occupy the left-half space inside the droplet. We also notice that the flows are relatively weak and no backward flows occurs in the region adjacent to the front end of the droplet. For $\theta^{e q} \geq 90^{\circ}$, two pairs of vortices are clearly visible inside and outside the droplet. The left vortex inside the droplet rotates clockwise while the right one rotates in an opposite direction. A similar structure of vortices was also experimentally observed by Baroud et al. [5] in a microfluidic crossjunction, where the laser heating was locally applied to block the droplet motion by generating thermocapillary convection. The formation of these vortex/recirculation regions is attributed to the response of the viscous forces to the thermocapillary stresses. It can easily be explained by the isotherms surrounding the droplet, which are shown in the right plane of Fig. 7, where the temperature value is labeled on each contour. For all contact angles, the left segment of the droplet interface is always exposed to an anticlockwise temperature gradient, leading to the vortex near the left meniscus inside the droplet rotating clockwise because the Marangoni stresses cause the fluid to flow away from regions of low interfacial tension (high temperature). For hydrophilic surfaces, the temperature gradient is anticlockwise (e.g., $\theta^{e q}=$ $30^{\circ}$ ) or very small (e.g., $\theta^{e q}=60^{\circ}$ ) along the right segment of the droplet 


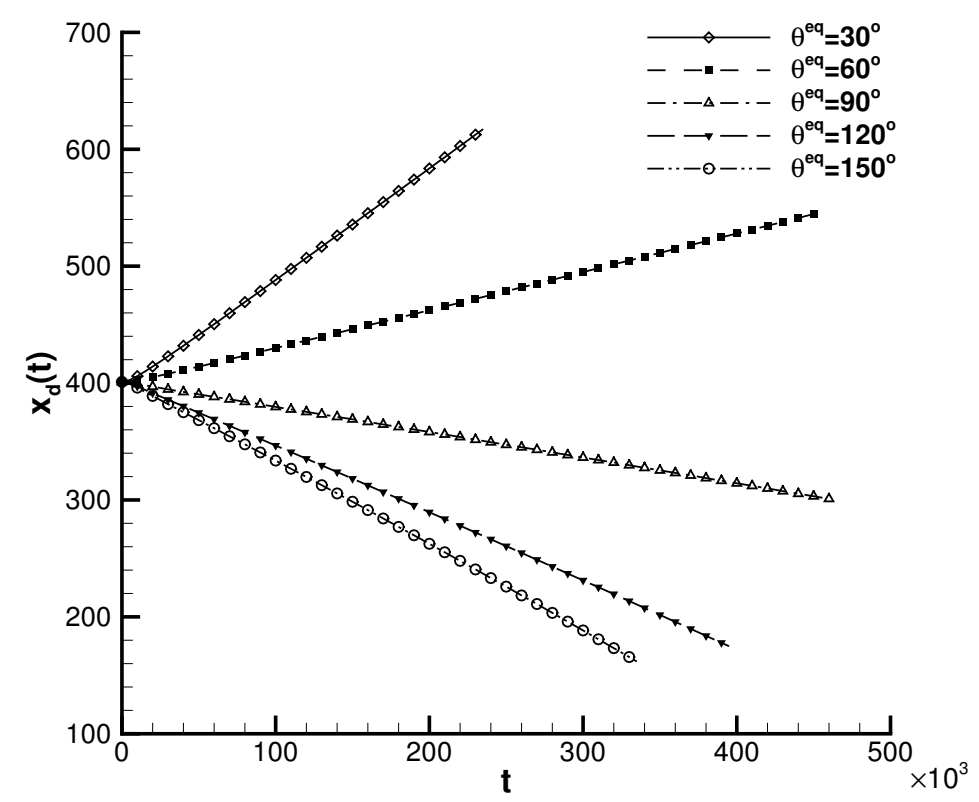

Figure 8: Time evolution of the $x$-coordinate of droplet centroid for $M=1$ at various contact angles.

interface due to a low curvature, and thus one cannot see the second internal vortex formed near the right meniscus. For neutral and hydrophobic surfaces, the high interface curvature causes different temperature gradients at the left and right segments of the droplet interface, inducing a pair of counterrotating vortices inside the droplet: the left vortex is bigger in size and stronger in intensity due to higher temperature gradient along the droplet interface.

In order to quantify the effect of surface wettability on droplet motion, Fig. 8 plots the time evolution of the $x$-coordinate of droplet centroid for $M=1$ at various $\theta^{e q}$, calculated by

$$
x_{d}(t)=\frac{\int_{V} \rho^{N} x \mathrm{~d} V}{\int_{V} \rho^{N} \mathrm{~d} V}=\frac{\sum_{\mathbf{x}} x(\mathbf{x}, t) \rho^{N}(\mathbf{x}, t)}{\sum_{\mathbf{x}} \rho^{N}(\mathbf{x}, t)}, \quad \text { where } \rho^{N}>0 .
$$

Obviously, $x_{d}$ varies linearly with the time for each $\theta^{e q}$, suggesting that the droplet migrates on the substrate at a constant velocity. For $\theta^{e q}<90^{\circ}$ the droplet migrates in the positive $x$-direction (i.e., from the hot to cold ends), whereas for $\theta^{e q} \geq 90^{\circ}$ it moves reversely. This result is consistent with the previous experimental observation reported in Song et al. [15] that the 


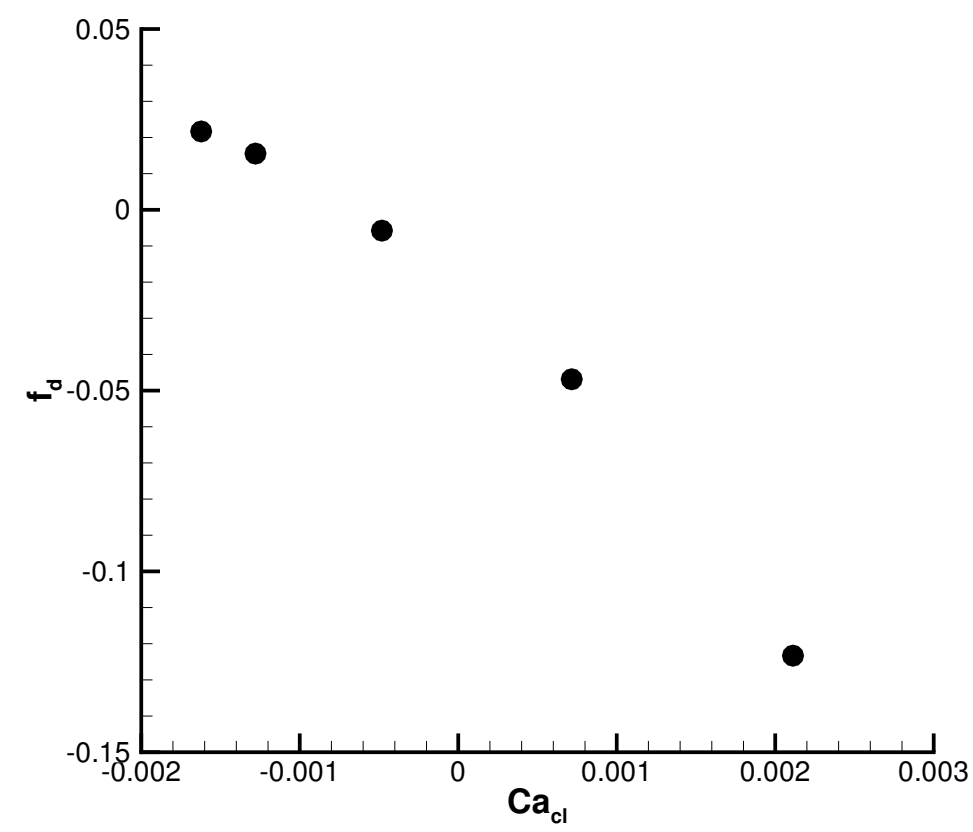

Figure 9: Dimensionless driving force $f_{d}$ acting on the moving droplet versus the contact-line capillary number $C a_{c l}$ at various $\theta^{e q}$ for $M=1$.

direction of droplet migration is opposite for the contact angles smaller and larger than $90^{\circ}$. By differentiating $x_{d}$ with respect to time, we obtain that, the migration velocities are $9.5 \times 10^{-4}, 3.22 \times 10^{-4},-2.17 \times 10^{-4},-5.76 \times 10^{-4}$ and $-7.3 \times 10^{-4}$ for $\theta^{e q}=30^{\circ}, 60^{\circ}, 90^{\circ}, 120^{\circ}$ and $150^{\circ}$, respectively. This indicates that more hydrophilicity or hydrophobicity favors to enhance the magnitude of migration velocity. It is therefore not surprising that the droplet can climb up an inclined superhydrophilic substrate as a result of thermally induced Marangoni stresses, against the action of gravity [18].

Table 2: Dynamic contact angles and contact angle hysteresis of a liquid droplet at various $\theta^{e q}$ for $M=1$. All angles are shown in degrees.

\begin{tabular}{cccccc}
\hline \hline$\theta^{e q}$ & 30 & 60 & 90 & 120 & 150 \\
\hline$\theta_{A}$ & 36.33 & 64.43 & 89.64 & 115.19 & 144.34 \\
$\theta_{R}$ & 34.34 & 63.95 & 89.14 & 113.66 & 142.01 \\
$\left(\theta_{A}-\theta_{R}\right)$ & 1.99 & 0.47 & 0.49 & 1.34 & 2.33 \\
\hline \hline
\end{tabular}

Table 2 shows the dynamic contact angles and contact angle hysteresis at various $\theta^{e q}$ when the droplet motion reaches the steady state. Each of 
the dynamic contact angles is evaluated as the intersection angle between the droplet interface tangent and the solid surface. The tangent is calculated by the connecting line of two intersection points, which are produced by the interface with the solid surface (denoted as point A) and the interface with the adjacent grid layer (denoted as point B), respectively. Although a constant contact angle (equal to $\theta^{e q}$ ) is imposed through Eq. (26) at the solid surface, the measured dynamic contact angle is different from $\theta^{e q}$ because the contact line motion can cause the relative position of point $B$ (with respect to point A) to change, deviating from the one in static state. It is observed that the advancing and receding contact angles are both greater than $\theta^{e q}$ for a hydrophilic surface, but smaller than $\theta^{e q}$ for a hydrophobic or neutral surface. We can also see that the difference between the dynamic contact angles and $\theta^{e q}$ is negligibly small for a neutral surface, suggesting that the capillary flow is not induced inside the droplet. Thus, the thermocapillary flow dominates the droplet dynamic behavior in this case. In addition, $\theta_{A}$ is found to be greater than $\theta_{R}$ for all contact angles considered. However, the contact angle hysteresis is insignificanttypically in the range of $\left(\theta_{A}-\theta_{R}\right) \leq 2.33^{\circ}$, which is consistent with the previous experimental and numerical findings [12,67]. Further, Fig. 9 shows the variation of the dimensionless driving force $\left(f_{d}\right)$ with the contact-line capillary number $\left(C a_{c l}\right)$ in the steady state. The dimensionless driving force is defined as $f_{d}=\frac{\sigma_{l} \cos \theta_{l}-\sigma_{r} \cos \theta_{r}}{\sigma_{r e f}}$, where the subscripts 'l' and ' $r$ ' denote the three-phase contact points on the left and right, respectively. The contact-line capillary number is defined as $C a_{c l}=\frac{u_{c l} \rho \nu_{B}}{\sigma_{r e f}}$, where $u_{c l}$ is the contact-line velocity. We can observe that $f_{d}$ decreases monotonously with an increase in $C a_{c l}$. However, $\left|f_{d}\right|$ increases with $\left|C a_{c l}\right|$ on either hydrophilic $\left(C a_{c l}>0\right)$ or hydrophobic $\left(C a_{c l}<0\right)$ surface.

For the thermocapillary migration of a droplet or bubble in an infinite medium, the viscosity ratio was found to strongly affect its migration velocity [1]. It is believed that the influence of viscosity ratio is more complicated for a droplet adhering to a solid surface due to the presence of fluid-surface interactions. Therefore, we examine the influence of viscosity ratio at three different contact angles, i.e., $\theta^{e q}=30^{\circ}, 90^{\circ}$ and $150^{\circ}$. For each $\theta^{e q}$, different viscosity ratios are achieved by adjusting the viscosity of dispersed phase while keeping the viscosity of continuous phase fixed. Fig. 10 shows the 

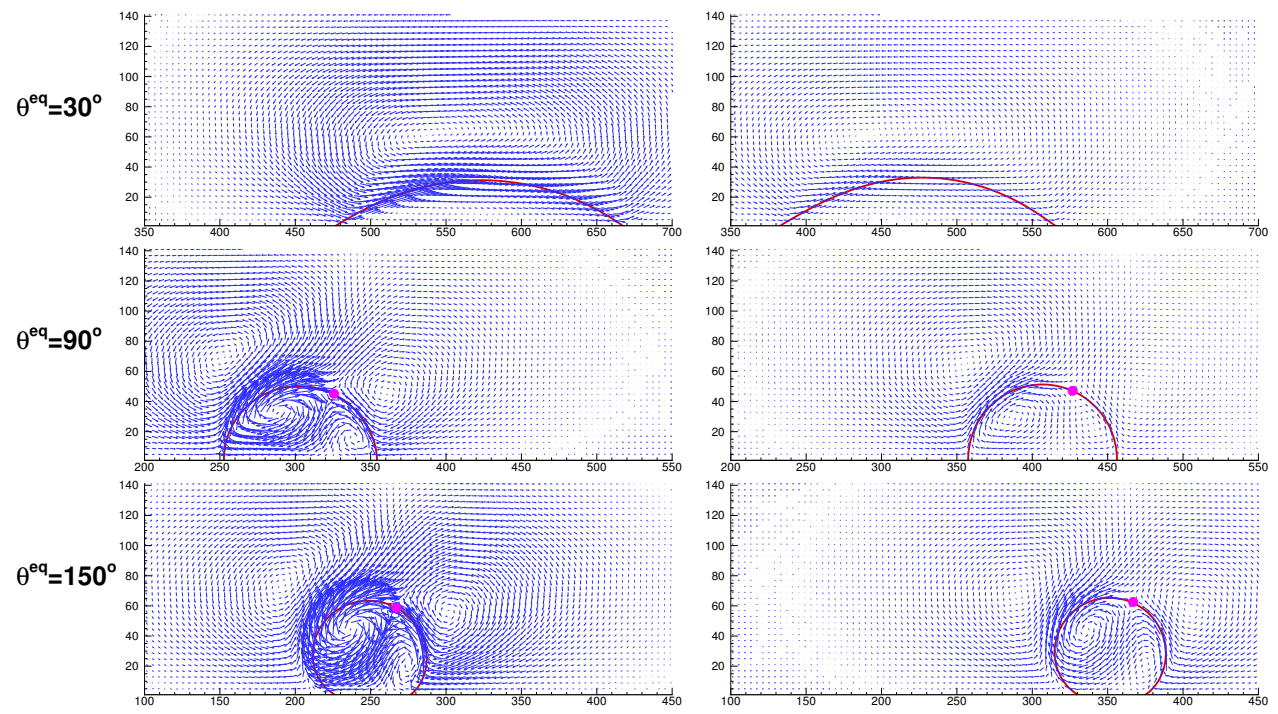

(a) $\mathrm{M}=0.35$

(b) $M=3.5$

Figure 10: (Color online) Droplet shape, position and velocity field surrounding the moving droplet for (a) $M=0.35$ and (b) $M=3.5$ at the contact angles of $30^{\circ}, 90^{\circ}$ and $150^{\circ}$. The red lines are zero contours of $\rho^{N}$, and the blue lines with arrows are the velocity vectors. The pink filled circles indicate the positions of stagnation point. 
shape and position of the droplet and its surrounding velocity field for the viscosity ratios of 0.35 and 3.5 at $t=1.5 \times 10^{5}$. For a fixed $\theta^{e q}$, one cannot see any significant change in droplet shape with $M$, and the structure of vortices (i.e., velocity field) exhibits similar characteristics to the case of $M=1$ as shown in Fig. 7. However, it is observed that the vortices significantly weaken with an increase in $M$. For all of the viscosity ratios under consideration, the droplet moves toward cooler regions on the hydrophilic surface, i.e., $\theta^{e q}=30^{\circ}$, and toward warmer regions on the hydrophobic surface, i.e., $\theta^{e q}=150^{\circ}$. On the neutral surface, the direction of droplet motion exhibits dependence on the value of $M$ : the droplet moves toward the warmer regions for low $M(M \leq 1)$, whereas it moves toward the cooler regions for high $M$ $(M=3.5)$. When the asymmetric vortex pair is formed inside the droplet, one can observe a stagnation point (indicated by the pink filled circles in Fig. 10) at the droplet interface, near the midplane of the droplet and closer to the cool end. At the stagnation point, the velocity is zero and the temperature is lowest compared to other positions at the interface. To capture the variation of stagnation point with $M$ for a moving droplet, we define a dimensionless position as $X_{s}=\frac{x_{r}-x_{s}}{x_{r}-x_{l}}$, where the subscript 's' refers to the stagnation point. As $M$ is varied from 0.35 to 3.5 , we find that $X_{S}$ decreases from 0.3 to 0.285 and from 0.244 to 0.199 for $\theta^{e q}=90^{\circ}$ and $150^{\circ}$, respectively. This indicates that increasing (decreasing) the migration velocity directed to the cooler (warmer) end leads to the stagnation point shifting from near the midplane to the cooler end.

Table 3: Dynamic contact angles and contact angle hysteresis of a liquid droplet at $M=$ 0.35 and 3.5 for $\theta^{e q}=30^{\circ}, 90^{\circ}$ and $150^{\circ}$. All angles are shown in degrees.

\begin{tabular}{c|ccc|ccc}
\hline \hline \multirow{2}{*}{$\theta^{e q}$} & \multicolumn{3}{|c|}{$M=0.35$} & \multicolumn{3}{c}{$M=3.5$} \\
\cline { 2 - 7 } & $\theta_{A}$ & $\theta_{R}$ & $\left(\theta_{A}-\theta_{R}\right)$ & $\theta_{A}$ & $\theta_{R}$ & $\left(\theta_{A}-\theta_{R}\right)$ \\
\hline 30 & 36.46 & 34.29 & 2.16 & 35.91 & 34.68 & 1.23 \\
90 & 89.93 & 88.58 & 1.36 & 89.52 & 89.45 & 0.06 \\
150 & 144.38 & 141.26 & 3.12 & 144.08 & 142.87 & 1.21 \\
\hline \hline
\end{tabular}

Fig. 11 illustrates the time evolution of the $x$-coordinate of droplet centroid for the viscosity ratios of $0.35,1$, and 3.5 at $\theta^{e q}=30^{\circ}, 90^{\circ}$ and $150^{\circ}$. For all cases, the droplet migrates at an approximately constant velocity, but the migration velocity is strongly dependent on the viscosity ratio and the contact angle. For each fixed $\theta^{e q}$, the migration velocity decreases with increasing viscosity ratio, which is in agreement with the theory of Young et 


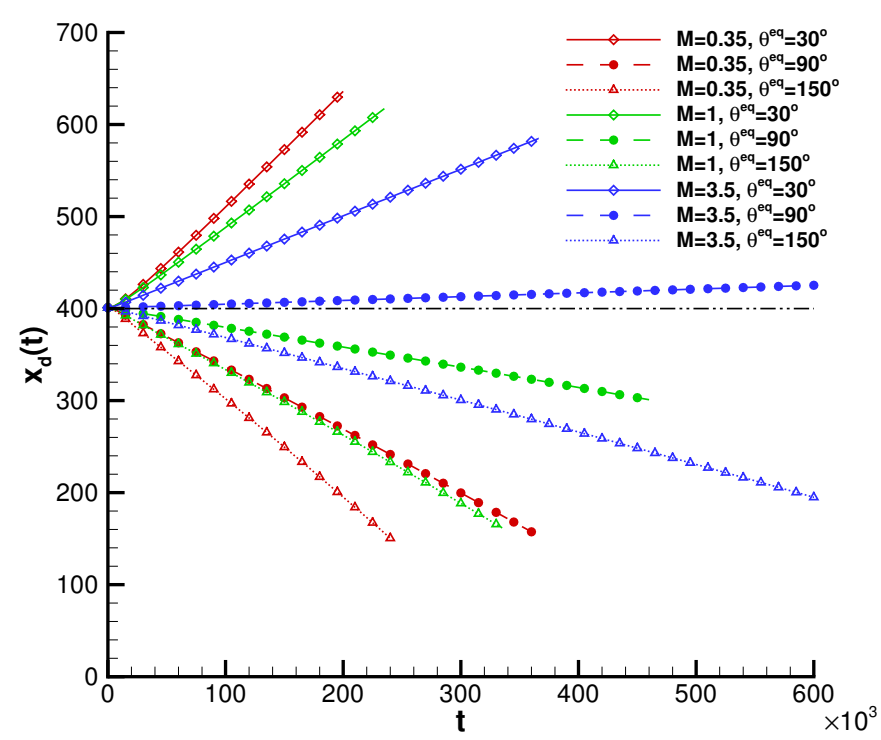

Figure 11: (Color online) Time evolution of the $x$-coordinate of droplet centroid for $M=$ $0.35,1$ and 3.5 at different contact angles. The dash-dot-dot line represents the initial $x$-coordinate of droplet centroid. 
al. [1] that quantifies the thermocapillary migration of a droplet/bubble in an infinite domain. Additionally, it is interesting to find that the viscosity ratio can also change the direction of droplet migration for $\theta^{e q}=90^{\circ}$. Although a high viscosity ratio causes the droplet to migrate toward the cooler regions on the neutral surface, the migration velocity is extremely low (e.g., the case of $M=3.5$ and $\theta^{e q}=90^{\circ}$ in Fig. 11). In a viewpoint of practical application, it is useless to use the thermocapillary flows for controlling a more viscous droplet on a neutral surface since the migration velocity is too weak to counteract effectively the external flows. For each fixed $M$, the droplet generally migrates faster on the hydrophilic surface (i.e., $\theta^{e q}=30^{\circ}$ ) than on the hydrophobic surface (i.e., $\theta^{e q}=150^{\circ}$ ). Finally, we find that the viscosity ratio and the contact angle can also affect the dynamic contact angles and contact angle hysteresis, which is shown in Table 3. For all cases, the advancing and receding contact angles both deviate from the equilibrium contact angle. Specifically, $\theta_{A}$ and $\theta_{R}$ are both smaller than $\theta^{e q}$ for $\theta^{e q}=90^{\circ}$ and $150^{\circ}$ but greater than $\theta^{e q}$ for $\theta^{e q}=30^{\circ}$. Also, $\theta_{A}$ is always larger than $\theta_{R}$, resulting in a positive contact angle hysteresis. For a fixed $\theta^{e q}$, it can be observed in Table 3 that increasing $M$ can lead to an increase in $\theta_{R}$ and a decrease in $\theta_{A}$. For each fixed $M$, the contact angle hysteresis first decreases and then increases as $\theta^{e q}$ increases, and the lowest hysteresis occurs at $\theta^{e q}=90^{\circ}$.

To further investigate the capability of the present model in simulating two-phase fluids with large viscosity differences, i.e., high or low viscosity ratios, we simulate the thermocapillary migration of a droplet on a horizontal solid surface subject to a uniform temperature gradient at $\theta^{e q}=150^{\circ}$ for $M=\frac{1}{100}, \frac{1}{500}$, and $\frac{1}{1000}$. The viscosity of blue fluid is kept at 0.3 , and the viscosity of red fluid is varied to obtain different $M$. The size of computational domain, and the initial location and radius of droplet are kept the same as the above simulations. Re, $C a, M a$, and $\chi$ are fixed at $0.333,1.33 \times 10^{-2}, 1$, and 1 , respectively. We find that the numerical simulations are still stable for $M=\frac{1}{100}$ and $\frac{1}{500}$. Fig. 12 gives the droplet shape, position and velocity field surrounding the moving droplet at $t=3 \times 10^{5}$ for (a) $M=\frac{1}{100}$ and (b) $M=\frac{1}{500}$. Note that the droplet migration has reached the steady state at this time. It can be clearly seen that the droplet migrates toward the warmer regions for both viscosity ratios, but migrates faster for a lower viscosity ratio, consistent with our observations in Fig. 11. However, numerical instability is observed when the viscosity ratio is decreased to $\frac{1}{1000}$. This might 
(a)

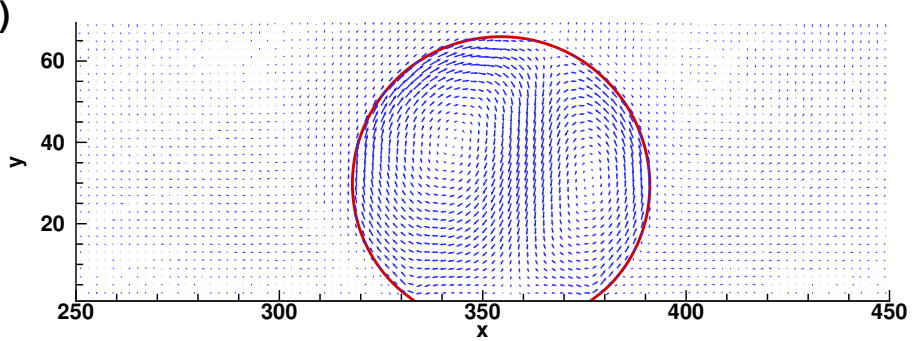

(b)

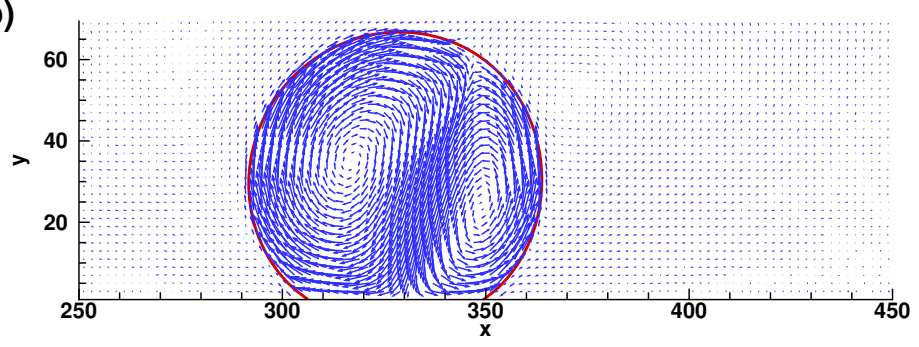

Figure 12: (Color online) Droplet shape, position and velocity field surrounding the moving droplet for (a) $M=\frac{1}{100}$ and (b) $M=\frac{1}{500}$ at the contact angle of $150^{\circ}$. The red lines are zero contours of $\rho^{N}$, and the blue lines with arrows are the velocity vectors.

be caused by low $\nu_{R}$, whose value is as low as $3 \times 10^{-4}$.

\section{Conclusions}

A lattice Boltzmann color-fluid model has been developed to simulate immiscible thermocapillary flows with the presence of fluid-surface interactions. Following our previous work [40], the CSF concept is employed to model the interfacial tension forces and the Marangoni stresses because of the gradient of interfacial tension, and the recoloring algorithm proposed by LatvaKokko and Rothman [44] is used to maintain the fluid-fluid interface and overcome the lattice pinning problem. At the solid surface, our recently developed wetting boundary condition [46] is introduced to account for fluidsurface interactions, which can ensure mass conservation for each fluid and suppress spurious velocities at the contact line. An additional convectiondiffusion equation is also solved using the passive scalar approach to obtain temperature field, which is related to the interfacial tension by an equation of state.

The lattice Boltzmann color-fluid model is first validated by simulations of equilibrium contact angle and dynamic capillary intrusion. This model is 
then used to simulate the transient thermocapillary migration of a microfluidic droplet on a horizontal solid surface subject to a uniform temperature gradient. Depending on the surface wettability, we numerically demonstrate for the first time that the droplet migration can undergo two different states, i.e., the droplet migrates toward the cooler regions on the hydrophilic surface and toward the warmer regions on the hydrophobic surface, which is consistent with the theoretical analysis and experimental observations $[66,15]$. For the droplet migration on hydrophilic surfaces, only one vortex is formed inside the droplet near the rear meniscus; whereas for the droplet migration on neutral and hydrophobic surfaces, two counter-rotating thermocapillary vortices are observed inside the droplet with the one on the warm side always greater in size. Increasing the viscosity ratio of droplet to carrier fluid leads to a reduction in migration velocity due to weakening of thermocapillary convection. The viscosity ratio can also affect the direction of droplet migration on the neutral surface, e.g., the droplet migrates toward the warmer regions for $M \leq 1$ but toward the cooler regions for $M=3.5$. When the droplet migrates on the solid surface, the advancing contact angle is always larger than the receding contact angle, no matter what the viscosity ratio and contact angle, leading a positive contact angle hysteresis. For each fixed $M$, the contact angle hysteresis and the magnitude of migration velocity first decreases and then increases with an increase in $\theta^{e q}$, and their minimum values both occur at $\theta^{e q}=90^{\circ}$.

\section{Acknowledgments}

YHZ thanks the UK's Royal Academy of Engineering (RAE) and the Leverhulme Trust for the award of a RAE/Leverhulme Trust Senior Research Fellowship.

\section{References}

[1] N. Young, J. Goldstein, M. Block, The motion of bubbles in a vertical temperature gradient, J. Fluid Mech. 6 (1959) 350-356.

[2] R. S. Subramanian, R. Balasubramaniam, The Motion of Bubbles and Drops in Reduced Gravity, Cambridge: Cambridge University Press, 2001.

[3] Z. J. Jiao, X. Y. Huang, N.-T. Nguyen, Manipulation of a droplet in 
a planar channel by periodic thermocapillary actuation, J. Micromech. Microeng. 18 (2008) 045027.

[4] M.-C. Liu, J.-G. Wu, M.-F. Tsai, W.-S. Yu, P.-C. Lin, I.-C. Chiu, H.-A. Chin, I.-C. Cheng, Y.-C. Tung, J.-Z. Chen, Two dimensional thermoelectric platforms for thermocapillary droplet actuation, RSC Adv. 2 (2012) 1639-1642.

[5] C. Baroud, J.-P. Delville, F. Gallaire, R. Wunenburger, Thermocapillary valve for droplet production and sorting, Phys. Rev. E 75 (2007) 046302.

[6] M. L. Cordero, D. R. Burnham, C. N. Baroud, D. McGloin, Thermocapillary manipulation of droplets using holographic beam shaping: microfluidic pin ball, Appl. Phys. Lett. 93 (2008) 034107.

[7] C. Baroud, M. Robert de Saint Vincent, J.-P. Delville, An optical toolbox for total control of droplet microfluidics, Lab Chip 7 (2007) 10291033.

[8] M. Robert de Saint Vincent, R. Wunenburger, J.-P. Delville, Laser switching and sorting for high speed digital microfluidics, Appl. Phys. Lett. 92 (2008) 154105.

[9] H. Liu, A. J. Valocchia, Y. Zhang, Q. Kang, Lattice Boltzmann phasefield modeling of thermocapillary flows in a confined microchannel, J. Comput. Phys. 256 (2014) 334-356.

[10] V. Pratap, N. Moumen, R. S. Subramanian, Thermocapillary motion of a liquid drop on a horizontal solid surface, Langmuir 24 (2008) 51855193.

[11] J. B. Brzoska, F. Brochard-Wyart, F. Rondelez, Motions of droplets on hydrophobic model surfaces induced by thermal gradients, Langmuir 9 (1993) 2220-2224.

[12] J. Z. Chen, S. M. Troian, A. A. Darhuber, S. Wagner, Effect of contact angle hysteresis on thermocapillary droplet actuation, J. Appl. Phys. 97 (2005) 014906.

[13] Z. Jiao, X. Huang, N.-T. Nguyen, P. Abgrall, Thermocapillary actuation of droplet in a planar microchannel, Microfluid. Nanofluid. 5 (2008) 205214. 
[14] Y.-T. Tseng, F.-G. Tseng, Y.-F. Chen, C.-C. Chieng, Fundamental studies on micro-droplet movement by Marangoni and capillary effects, Sensors and Actuators A: Physical 114 (2004) 292 - 301.

[15] C. Song, K. Kim, K. Lee, H. K. Pak, Thermochemical control of oil droplet motion on a solid substrate, Applied Physics Letters 93 (8) (2008) 084102.

[16] M. L. Ford, A. Nadim, Thermocapillary migration of an attached drop on a solid surface, Phys. Fluids 6 (9) (1994) 3183-3185.

[17] M. K. Smith, Thermocapillary migration of a two-dimensional liquid droplet on a solid surface, J. Fluid Mech. 294 (1995) 209-230.

[18] G. Karapetsas, K. C. Sahu, O. K. Matar, Effect of contact line dynamics on the thermocapillary motion of a droplet on an inclined plate, Langmuir 29 (2013) 8892-8906.

[19] J. H. Snoeijer, B. Andreotti, Moving contact lines: Scales, regimes, and dynamical transitions, Annu. Rev. Fluid Mech. 45 (2013) 269-292.

[20] C. Hirt, B. Nichols, Volume of fluid (VOF) method for the dynamics of free boundaries, J. Comput. Phys. 39 (1981) 201-225.

[21] D. Gueyffier, J. Li, A. Nadim, R. Scardovelli, S. Zaleski, Volume-offluid interface tracking with smoothed surface stress methods for threedimensional flows, J. Comput. Phys. 152 (2) (1999) 423-456.

[22] M. Sussman, E. Fatemi, P. Smereka, S. Osher, An improved level set method for incompressible two-phase flows, Comput. Fluids 27 (1998) 663-680.

[23] S. Osher, R. P. Fedkiw, Level sets methods and dynamic implicit surfaces, Springer, 2003.

[24] W. Shyy, R. W. Smith, H. S. Udaykumar, M. M. Rao, Computational fluid dynamics with moving boundaries, Taylor \& Francis, Washington, DC, 1996.

[25] L. M. Hocking, A moving fluid interface. Part 2. the removal of the force singularity by a slip flow, J. Fluid Mech. 79 (1977) 209-229. 
[26] H.-Y. Chen, D. Jasnow, J. Viñals, Interface and contact line motion in a two phase fluid under shear flow, Phys. Rev. Lett. 85 (2000) 1686-1689.

[27] S. Chen, G. D. Doolen, Lattice Boltzmann method for fluid flows, Annu. Rev. Fluid Mech. 30 (1) (1998) 329-364.

[28] A. K. Gunstensen, D. H. Rothman, S. Zaleski, G. Zanetti, Lattice Boltzmann model of immiscible fluids, Phys. Rev. A 43 (8) (1991) 4320-4327.

[29] I. Halliday, R. Law, C. M. Care, A. Hollis, Improved simulation of drop dynamics in a shear flow at low Reynolds and capillary number, Phys. Rev. E 73 (5) (2006) 056708.

[30] T. Reis, T. N. Phillips, Lattice Boltzmann model for simulating immiscible two-phase flows, Journal of Physics A: Mathematical and Theoretical 40 (14) (2007) 4033-4053.

[31] H. Liu, A. J. Valocchi, Q. Kang, Three-dimensional lattice Boltzmann model for immiscible two-phase flow simulations, Phys. Rev. E 85 (2012) 046309.

[32] M. R. Swift, E. Orlandini, W. R. Osborn, J. M. Yeomans, Lattice Boltzmann simulations of liquid-gas and binary fluid systems, Phys. Rev. E 54 (5) (1996) 5041-5052.

[33] H. Zheng, C. Shu, Y. Chew, A lattice Boltzmann model for multiphase flows with large density ratio, J. Comput. Phys. 218 (1) (2006) 353-371.

[34] T. Lee, L. Liu, Lattice Boltzmann simulations of micron-scale drop impact on dry surfaces, J. Comput. Phys. 229 (20) (2010) 8045-8063.

[35] X. Shan, H. Chen, Lattice Boltzmann model for simulating flows with multiple phases and components, Phys. Rev. E 47 (3) (1993) 1815-1819.

[36] X. Shan, H. Chen, Simulation of nonideal gases and liquid-gas phase transitions by the lattice Boltzmann equation, Phys. Rev. E 49 (1994) 2941-2948.

[37] M. Sbragaglia, R. Benzi, L. Biferale, S. Succi, K. Sugiyama, F. Toschi, Generalized lattice Boltzmann method with multirange pseudopotential, Phys. Rev. E 75 (2007) 026702. 
[38] X. He, S. Chen, R. Zhang, A lattice Boltzmann scheme for incompressible multiphase flow and its application in simulation of Rayleigh-Taylor instability, J. Comput. Phys. 152 (2) (1999) 642-663.

[39] J. Zhang, Lattice Boltzmann method for microfluidics: models and applications, Microfluid. Nanofluid. 10 (1) (2011) 1-28.

[40] H. Liu, Y. Zhang, A. J. Valocchi, Modeling and simulation of thermocapillary flows using lattice Boltzmann method, J. Comput. Phys. 231 (12) (2012) 4433-4453.

[41] H. Liu, A. J. Valocchi, Y. Zhang, Q. Kang, A phase-field-based latticeBoltzmann finite-difference model for simulating thermocapillary flows, Phys. Rev. E 87 (2013) 013010.

[42] S. V. Lishchuk, C. M. Care, I. Halliday, Lattice Boltzmann algorithm for surface tension with greatly reduced microcurrents, Phys. Rev. E 67 (2003) 036701.

[43] J. U. Brackbill, D. B. Kothe, C. Zemach, A continuum method for modeling surface tension, Journal of Computational Physics 100 (2) (1992) $335-354$.

[44] M. Latva-Kokko, D. H. Rothman, Diffusion properties of gradient-based lattice Boltzmann models of immiscible fluids, Phys. Rev. E 71 (2005) 056702 .

[45] Y. Peng, C. Shu, Y. Chew, Simplified thermal lattice Boltzmann model for incompressible thermal flows, Phys. Rev. E 68 (2003) 026701.

[46] Y. Ba, H. Liu, J. Sun, R. Zheng, Color-gradient lattice Boltzmann model for simulating droplet motion with contact-angle hysteresis, Phys. Rev. E 8888 (2013) 043306.

[47] H. Ding, P. D. M. Spelt, Wetting condition in diffuse interface simulations of contact line motion, Phys. Rev. E 75 (2007) 046708.

[48] A. P. Hollis, T. J. Spencer, I. Halliday, C. M. Care, Dynamic wetting boundary condition for continuum hydrodynamics with multicomponent lattice Boltzmann equation simulation method, IMA J. Appl. Math. 76 (2011) 726-742. 
[49] Y. H. Qian, D. D'Humières, P. Lallemand, Lattice BGK models for Navier-Stokes equation, Europhys. Lett. 17 (1992) 479-484.

[50] Z. Guo, C. Zheng, B. Shi, Discrete lattice effects on the forcing term in the lattice Boltzmann method, Phys. Rev. E 65 (2002) 046308.

[51] Y. Q. Zu, S. He, Phase-field-based lattice Boltzmann model for incompressible binary fluid systems with density and viscosity contrasts, Phys. Rev. E 87 (2013) 043301.

[52] S. Leclaire, M. Reggio, J.-Y. Trépanier, Numerical evaluation of two recoloring operators for an immiscible two-phase flow lattice Boltzmann model, Applied Mathematical Modelling 36 (2012) 2237-2252.

[53] I. Halliday, A. P. Hollis, C. M. Care, Lattice Boltzmann algorithm for continuum multicomponent flow, Phys. Rev. E 76 (2007) 026708.

[54] H. Liu, Y. Zhang, Droplet formation in microfluidic cross-junctions, Phys. Fluids 23 (8) (2011) 082101.

[55] H. Ding, P. D. M. Spelt, Inertial effects in droplet spreading: a comparison between diffuse-interface and level-set simulations, J. Fluid Mech. 576 (2007) 287-296.

[56] C. M. Pooley, K. Furtado, Eliminating spurious velocities in the freeenergy lattice Boltzmann method, Phys. Rev. E 77 (2008) 046702.

[57] X. Shan, Analysis and reduction of the spurious current in a class of multiphase lattice Boltzmann models, Phys. Rev. E 73 (2006) 047701.

[58] T. Lee, P. F. Fischer, Eliminating parasitic currents in the lattice boltzmann equation method for nonideal gases, Phys. Rev. E 74 (4) (2006) 046709 .

[59] Z. Yu, L.-S. Fan, Multirelaxation-time interaction-potential-based lattice Boltzmann model for two-phase flow, Phys. Rev. E 82 (2010) 046708.

[60] H. Liu, Y. Zhang, Lattice Boltzmann simulation of droplet generation in a microfluidic cross-junction, Commun. Comput. Phys. 9 (5) (2011) 1235-1256. 
[61] C. M. Pooley, H. Kusumaatmaja, J. M. Yeomans, Contact line dynamics in binary lattice Boltzmann simulations, Phys. Rev. E 78 (5) (2008) 056709 .

[62] E. W. Washburn, The dynamics of capillary flow, Phys. Rev. 17 (1921) 273-283.

[63] F. Diotallevi, L. Biferale, S. Chibbaro, A. Lamura, G. Pontrelli, M. Sbragaglia, S. Succi, F. Toschi, Capillary filling using lattice Boltzmann equations: The case of multi-phase flows, Eur. Phys. J. Special Topics 166 (2009) 111-116.

[64] C. Pooley, H. Kusumaatmaja, J. Yeomans, Modelling capillary filling dynamics using lattice Boltzmann simulations, Eur. Phys. J. Special Topics 171 (1) (2009) 63-71.

[65] C.-H. Liu, K.-H. Lin, H.-C. Mai, C.-A. Lin, Thermal boundary conditions for thermal lattice Boltzmann simulations, Comput. Math. Appl. 59 (2010) 2178-2193.

[66] T. S. Sammarco, M. A. Burns, Thermocapillary pumping of discrete drops in microfabricated analysis devices, AIChE J. 45 (1999) 350-366.

[67] H.-B. Nguyen, J.-C. Chen, A numerical study of thermocapillary migration of a small liquid droplet on a horizontal solid surface, Phys. Fluids 22 (2010) 062102. 